\title{
Terapia Vibro-Oscilatória na Reabilitação da Função RespiratóRIa da Pessoa com Atelectasia Pulmonar: Relato de Caso
}

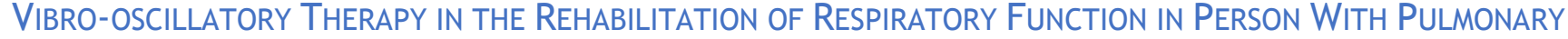 \\ ATELECTASIS: CASE REPORT
}

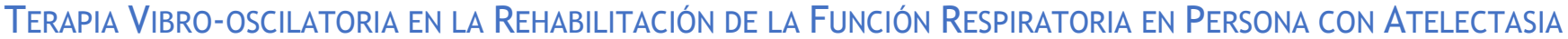 PULMONAR: REPORTE DE CASO}

DOI 10.33194/rper.2022.198

Data de Receção: 2021-11-15 Data de Aceitação: 2021-12-31 Data de publicação on-line: 2022-01-16

\author{
Sérgio Vaz ${ }^{1}$; ; Ana Sofia Loureiro²; Ana Sofia Vilela ${ }^{3} \mathbb{D}$; Jacinta Martins ${ }^{4}$; Andreia Felix ${ }^{5}$ (D); André Novo ${ }^{6}$ (D) \\ ${ }^{1}$ Serviço de Pneumologia, Unidade de Reabilitação Respiratória, Centro Hospitalar de Trás-os-Montes e Alto Douro, Vila Real, \\ Portugal \\ ${ }^{2}$ Serviço de Cirurgia, Centro Hospitalar de Trás-os-Montes e Alto Douro, Vila Real, Portugal \\ ${ }^{3}$ Serviço de Medicina Intensiva, Centro Hospitalar de Trás-os-Montes e Alto Douro, Vila Real, Portugal \\ ${ }^{4}$ Escola Superior de Enfermagem Cruz Vermelha Portuguesa Alto Tâmega, Chaves, Portugal \\ ${ }^{5}$ Nursing Research, Innovation and Development Centre of Lisbon; Escola Superior de Enfermagem Cruz Vermelha Portuguesa Alto \\ Tâmega, Chaves, Portugal \\ ${ }^{6}$ Instituto Politécnico de Bragança, Bragança, Portugal' CINTESIS:NursID, Portugal
}

Autor correspondente: André Novo, andre@ipb.pt

\section{RESUMO}

Introdução: A pessoa com atelectasia pulmonar pode apresentar alterações significativas na função respiratória, sendo de carácter imprescindível uma intervenção especializada de reabilitação com os objetivos de potenciar a qualidade de vida da pessoa, otimizar a função respiratória, capacitar a pessoa e gerir os sintomas respiratórios.

Objetivo: Avaliar os ganhos sensíveis aos cuidados de enfermagem de reabilitação na otimização da função respiratória de uma pessoa com o diagnóstico clínico de atelectasia pulmonar com recurso a terapia vibrooscilatória.

Metodologia: Estudo descritivo do tipo relato de caso elaborado de acordo com as guidelines da CAse REports. Aplicado o processo de enfermagem com recurso ao Padrão Documental dos Cuidados da Especialidade de Enfermagem de Reabilitação. Pessoa do sexo masculino, com 73 anos de idade, admitido no internamento de pneumologia com alteração da função respiratória e capacidade de expetorar comprometida.

Resultados: Com a implementação do programa de intervenção de Enfermagem de Reabilitação evidenciaram-se ganhos na diminuição da dispneia, culminando com a alta da pessoa e reinserção na comunidade.

Conclusão: Com a sistematização e implementação de um plano de cuidados de enfermagem de reabilitação individualizado é possível registar a efetividade das intervenções no controlo e redução da dispneia da pessoa.

DESCRITORES: Atelectasia pulmonar, Reabilitação respiratória, Enfermagem de Reabilitação, Relato de caso

\section{ABSTRACT}

Introduction: A person with pulmonary atelectasis can present significant changes in respiratory function, and a specialized rehabilitation intervention is essential to enhance the person's quality of life, optimize respiratory function, enable the person and respiratory symptom management.

Objective: Evaluate the sensible gains of rehabilitation nursing care in optimizing the respiratory function of a person with a clinical diagnosis of pulmonary atelectasis using vibro-oscillatory therapy.

Methodology: Descriptive case report study prepared in accordance with the guidelines of the CAse REports. Applied the nursing process using the Documentary Standard of Care of the Rehabilitation Nursing Specialty. A 73 - 
year-old male, admitted to the pulmonology service with impaired respiratory function and impaired sputum capacity.

Results: With the implementation of the Rehabilitation Nursing intervention program, gains were evidenced in the reduction of dyspnea, culminating in the person's discharge and reintegration into community.

Conclusion: With the systematization and implementation of an individualized rehabilitation nursing care plan, it's possible to record the effectiveness of interventions in controlling and reducing the person's dyspnea.

DESCRIPTORS: Pulmonary Atelectasis; Breathing Exercises; Rehabilitation Nursing; Case Reports

\section{RESUMEN}

Introducción: Una persona con atelectasia pulmonar puede presentar cambios importantes en la función respiratoria, que pueden comprometer su calidad de vida, y una intervención rehabilitadora especializada es fundamental para mejorar la calidad de vida de la persona, optimizar la función respiratoria, capacitar a la persona y manejar los síntomas respiratorios.

Objetivo: Evaluar los beneficios sensibles de la atención de enfermería de rehabilitación en la optimización de la función respiratoria de una persona con un diagnóstico clínico de atelectasia pulmonar usando terapia vibrooscilatoria.

Metodología: Estudio descriptivo tipo reporte de caso elaborado de acuerdo con los lineamientos del CAse REports (CARE). Aplicó el proceso de enfermería utilizando el Estándar Documental de Atención de la Especialidad de Enfermería en Rehabilitación. Varón de 73 años, ingresado en el servicio de neumología con deterioro de la función respiratoria y deterioro de la capacidad de esputo.

Resultados: Con la implementación del programa de intervención de Enfermería de Rehabilitación se evidenciaron avances en la reducción de la disnea, culminando con el alta de la persona y su reintegración a la comunidad.

Conclusión: Con la sistematización e implementación de un plan de atención de enfermería rehabilitadora individualizada, es posible registrar la efectividad de las intervenciones en el control y reducción de la disnea de la persona.

DESCRIPTORES: Atelectasia pulmonar; Ejercicios de respiración; Enfermería de rehabilitación; Reporte de caso

\section{INTRODUÇÃO}

A atelectasia pulmonar é uma condição pulmonar caraterizada por um colapso reversível, parcial ou completo das vias aéreas de pequeno calibre ${ }^{(1)}$. Pode acometer um lobo, segmento ou todo o pulmão, causando diminuição da relação ventilação/perfusão $(\mathrm{V} / \mathrm{Q})^{(2)}$ e comprometendo as trocas gasosas de oxigénio e dióxido de carbono ${ }^{(1)}$.

A atelectasia pulmonar constitui uma das complicações respiratórias que podem contribuir para uma significativa morbilidade ${ }^{(3)}$. As consequências da atelectasia incluem a redução da compliance pulmonar, hipoxémia, aumento da resistência vascular pulmonar, pneumonia, podendo ainda conduzir a insuficiência respiratória aguda (IRA) ${ }^{(4)}$.

A atelectasia pode ocorrer por diferentes processos sendo comummente categorizada em obstrutiva, não obstrutiva, pós-operatória e atelectasia arredondada ${ }^{(3)}$. No caso da atelectasia obstrutiva, esta pode resultar de obstrução por um tampão mucoso, como observado em doenças como a asma, bronquite ou pessoas sob ventilação mecânica ${ }^{(2)}$. Frequentemente referida como atelectasia de reabsorção, sucede quando existe uma baixa relação Ventilação/Perfusão (V/Q) e a obstrução inibe parcial ou completamente a ventilação, mas a perfusão na área é preservada, mantendo-se a absorção do ar alveolar distal à obstrução, com consequente diminuição da tensão de nitrogénio alveolar e colapso das vias aéreas ${ }^{(4,5)}$.

Normalmente, a atelectasia é assintomática ${ }^{(3)}$. As manifestações clínicas são pouco específicas e a intensidade das mesmas depende da extensão e gravidade da área afetada. Podem estar presentes sintomas como cianose, tosse, expetoração, dispneia, taquipneia e sudorese ${ }^{(6)}$.

O exame físico do tórax pode revelar diminuição da excursão torácica, retrações intercostais e a auscultação pulmonar é marcada pela diminuição (ou abolição) dos sons respiratórios ou a presença de crepitações finas que podem desaparecer após respiração profunda ou tosse ${ }^{(6)}$.

A atelectasia geralmente não é evidente em telerradiografias convencionais do tórax até que seja significativa, podendo evidenciar opacificação pulmonar, hiperinsuflação compensatória no pulmão adjacente não atelectásico e nos casos mais graves de atelectasia unilateral, o deslocamento ipsilateral das estruturas intratorácicas adjacentes em direção à área $\operatorname{afetada}^{(7)}$. 
A gasometria arterial pode demonstrar hipoxemia arterial e alcalose respiratória. A pressão parcial venosa de dióxido de carbono $\left(\mathrm{PaCO}_{2}\right)$ é geralmente normal podendo, no entanto, ser menor, secundária ao aumento da ventilação/minuto que frequentemente acompanha a atelectasia ${ }^{(3)}$.

Antes de se selecionarem as possíveis estratégias de tratamento, é importante compreender o mecanismo, a causa e o significado funcional da atelectasia, já que não existe evidencia que alguma terapia isolada seja eficaz em todas as formas de atelectasia ${ }^{(5)}$, sendo recomendada a implementação de uma abordagem multifacetada com base nas necessidades individuais da pessoa ${ }^{(6)}$.

O tratamento deve visar a identificação da causa subjacente da doença e envolver medidas de suporte como exercícios de reexpansão pulmonar, broncodilatação, inaloterapia e oxigenoterapia ${ }^{(3,5)}$. As estratégias de tratamento descritas na literatura incluem geralmente a reeducação funcional respiratória como tratamento tradicional de primeira linha e utilização de pressões inspiratória e expiratória positivas ${ }^{(5)}$. 0 mecanismo subjacente a essas medidas é um aumento transitório na pressão transpulmonar que permita a reexpansão dos segmentos pulmonares colapsados ${ }^{(3)}$.

No caso da atelectasia obstrutiva por tampão mucoso, importa reverter a sua causa, quer pela diminuição da viscosidade do muco e aumento da depuração ciliar, quer pelo aumento do fluxo expiratório aplicando forças de cisalhamento e alta energia cinética ao muco aderido à parede das vias aéreas ${ }^{(8,9)}$.

Há uma grande variedade de técnicas ao dispor, no entanto a técnica aplicada deve ser adaptada à preferência da pessoa, levando em consideração que técnicas simples e rápidas podem estimular a adesão ao tratamento ${ }^{(10)}$.

A vibração consiste na aplicação de movimentos oscilatórios com os objetivos teóricos de alterar as propriedades reológicas do muco, induzir tosse por estimulação mecânica das vias aéreas e aumentar o pico de fluxo expiratório $(P F E)^{(11)}$. Na literatura é recorrentemente referido que a vibração da parede torácica de alta frequência na faixa de $11-15 \mathrm{~Hz}$ aumenta a taxa de depuração do muco, sendo a frequência ideal de $13 \mathrm{~Hz}^{(12)}$, a qual corresponde à frequência de batimento do sistema ciliar $^{(9)}$. Atualmente existem vários mecanismos de vibração, entre os quais dispositivos de oscilação da parede torácica de alta frequência e dispositivos de vibração percussiva intrapulmonar.

A oscilação da parede torácica de alta frequência é realizada através de um colete insuflado com pressão (1-10) e frequência variáveis $(5-20 \mathrm{~Hz})$. O fluxo de ar oscilatório na parede torácica externa gera aumentos transitórios no fluxo de ar expiratório nas vias aéreas, diminuição da viscoelasticidade do muco ${ }^{(8,13)}$, aumento da frequência do batimento ciliar ${ }^{(14,11)}$ e melhoria da distribuição da ventilação ${ }^{(15)}$. No entanto, o dispositivo não fornece meios de ventilar atrás das vias aéreas obstruídas, ao contrário de outros dispositivos oscilatórios que fornecem pressão expiratória positiva (PEP) $)^{(9)}$.

A vibração percussiva intrapulmonar tem sido progressivamente usada na pessoa com secreções excessivas ou para intervir na presença de atelectasias ${ }^{(16)}$. Esta consiste na administração de pequenos volumes correntes pulsáteis (entre $1 \mathrm{ml}$ e $300 \mathrm{ml}$ ) a alta frequência nas vias aéreas, criando um efeito global de vibropercussão interna dos pulmões sobrepondo-se à ventilação espontânea do paciente ${ }^{(17)}$. Tem como objetivos promover a limpeza das vias aéreas, recrutar unidades alveolares colapsadas (graças à PEP que permite uma distribuição mais homogénea do ar na árvore brônquica evitando a hiperinsuflação de territórios saudáveis) e melhorar as trocas gasosas ${ }^{(14,17,18)}$.

O presente relato de caso apresenta como questão de partida: "Na pessoa portadora de atelectasia pulmonar, qual a efetividade da intervenção do Enfermeiro Especialista em Enfermagem de Reabilitação, na melhoria da função respiratória com recurso a terapia vibro-oscilatória?

O presente relato de caso apresenta como objetivo geral: avaliar a efetividade da intervenção do Enfermeiro Especialista em Enfermagem de Reabilitação (EEER) na pessoa portadora de atelectasia pulmonar, com a aplicação de um programa de reabilitação respiratória com recurso a terapia vibro-oscilatória.

Identificam-se, ainda, os seguintes objetivos específicos:

- Identificar os ganhos sensíveis à reeducação funcional respiratória nos focos de Enfermagem ventilação e limpeza das vias aéreas;

- Descrever as intervenções do EEER nos respetivos focos.

\section{MATERIAL E MÉTODOS}

Trata-se de um estudo descritivo, do tipo Relato de Caso, construído com base nas guidelines da CAse REport (CARE), pois permite uma estruturação fomentadora de pensamento crítico, reflexão sobre as práticas de cuidados e objetivação dos ganhos na pessoa ${ }^{(19)}$.

O estudo descreve o caso de uma pessoa portadora de atelectasia pulmonar obstrutiva secundária a tampão mucoso, fazendo alusão ao desenho do programa de reabilitação respiratória implementado em contexto de internamento e os resultados na pessoa sensíveis ao programa instituído. A colheita de dados foi realizada mediante anamnese e consulta do processo clínico, com a aprovação da comissão de ética do Centro Hospitalar de Trás-osMontes e Alto Douro, E.P.E. 
O programa de reabilitação respiratória decorreu no mês de julho de 2021, com início no decurso do internamento.

A avaliação de Enfermagem de Reabilitação incidiu nos sinais e sintomas da doença respiratória: tosse e expetoração, avaliadas na sua eficácia e aspeto macroscópico. A função respiratória foi avaliada através do exame físico (auscultação), gasometria arterial e telerradiografia do tórax.

\section{APRESENTAÇÃO DO CASO}

\section{Anamnese}

0 presente caso retrata uma pessoa de 73 anos de idade, do género masculino, caucasiano e de nacionalidade portuguesa, casado e pai de dois filhos e atualmente reformado. Apresenta como antecedentes pessoais conhecidos: dislipidemia, hipertensão arterial, enfarte agudo do miocárdio, fibrilação auricular paroxística, neoplasia gástrica, carcinoma medular da tiróide, hipotiroidismo, fibrose pulmonar sequelar a pneumonite rádica, hérnia diafragmática e bronquiectasias. Regime medicamentoso habitual: Apixabana 5mg, 2 vezes/dia; Pantoprazol 20mg, $1 \mathrm{vez} /$ dia; Levotiroxina 137/125mg, em dias alternados; Bisoprolol 2,5mg, $1 \mathrm{vez} / \mathrm{dia}$; Atorvastatina $40 \mathrm{mg}, 1$ vez/dia; Amiodarona 100mg, 3 vezes/semana; Furosemida 20mg, 1 vez/dia.

Recorreu ao serviço de urgência no dia 19 de junho de 2021 por um quadro arrastado e progressivo de dispneia agravada desde há 3 meses, com sintomatologia associada de epigastralgias e vómitos. Realizada toracocentese evacuadora, com drenagem de $900 \mathrm{ml}$ de líquido citrino. No dia seguinte, por prostração com dessaturação

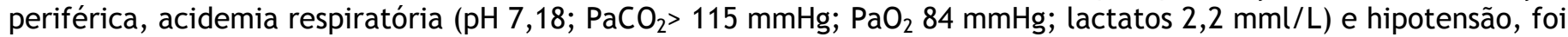
iniciada ventilação não invasiva e fluidoterapia. Nesse contexto, foi admitido no serviço de medicina intensiva com os problemas sequenciais de derrame pleural bilateral, insuficiência cardíaca descompensada, insuficiência respiratória mista e infeção respiratória por Klebsiella Pneumoniae. Foi transferido para o serviço de pneumologia a 15 de julho. A 2 de agosto de 2021, por atelectasia total à esquerda é referenciado para reabilitação funcional respiratória por Enfermagem de Reabilitação. Após implementação de programa individualizado de reeducação funcional respiratória teve alta a 18 de agosto de 2021.

\section{Avaliação de Enfermagem de Reabilitação}

A avaliação foi realizada com base no exame físico e exames complementares de diagnóstico, os quais são descritos no Quadro 1.

Com base na Avaliação Inicial de Enfermagem de Reabilitação, identificaram-se os diagnósticos e intervenções de enfermagem por forma a serem objetivados os ganhos na pessoa sensíveis aos cuidados de enfermagem de reabilitação.

Quadro 1 - Avaliação inicial

\begin{tabular}{|c|l|}
\hline Parâmetro & \multicolumn{1}{|c|}{ Caraterísticas } \\
\hline Telerradiografia torácica & Opacificação à esquerda - Derrame pleural de médio/grande volume \\
\hline Auscultação pulmonar & $\begin{array}{l}\text { Direita: murmúrio vesicular (MV) diminuído e fervores crepitantes. } \\
\text { Esquerda: MV abolido }\end{array}$ \\
\hline Tipo de respiração & Mista \\
Ritmo & Irregular \\
Amplitude & Diminuída \\
\hline Recrutamento músculos acessórios & Sim \\
\hline Muco & Presente \\
Cor & Amarela \\
\hline Viscoelasticidade & Alta \\
\hline Tosse & Ineficaz \\
\hline Pico de fluxo & $180 \mathrm{~L} / \mathrm{min}$ \\
\hline Aerossolterapia & Não \\
\hline Oxigenoterapia & $2 \mathrm{~L} / \mathrm{min}$ \\
\hline SpO2 & $93 \%$ \\
\hline Sinais Vitais & $95 / 66 \mathrm{mmHg}$ \\
TA & $80($ Bpm $)$ \\
FC & $20($ Ciclos $/ \mathrm{min})$ \\
\hline DR & 0 \\
\hline Temperatura & $36,0^{\circ}$ \\
\hline
\end{tabular}




\section{Diagnósticos de Enfermagem de Reabilitação}

Tendo como ponto de partida a avaliação inicial de Enfermagem de Reabilitação e de acordo com as necessidades e possibilidades da pessoa, foram identificados quatro diagnósticos de enfermagem relacionados com os focos "ventilação" e "expetorar", tendo como referencial a linguagem da Classificação Internacional para a Prática de Enfermagem $\left(\right.$ CIPE) ${ }^{\circledR}$ (2015), e tendo como base o padrão documental dos cuidados de Enfermagem da Especialidade de Enfermagem de Reabilitação(20) e o Guia Orientador da Boa Prática da Reabilitação Respiratória(21):

-Ventilação comprometida;

-Expetorar ineficaz;

-Potencial para melhorar conhecimento sobre técnica da tosse;

- Potencial para melhorar a capacidade para usar a técnica da tosse.

Os diagnósticos anteriormente identificados permitem sustentar o Programa de Reeducação Funcional Respiratória, apresentado de forma detalhada no Quadro 2.

Quadro 2 - Diagnósticos e Intervenções de enfermagem

\section{Diagnóstico de enfermagem: Ventilação comprometida}

\section{- Observar exame [gasometria arterial e telerradiografia do tórax];}

- Monitorizar oximetria periférica de oxigénio;

- Monitorizar os sinais vitais;

- Auscultar tórax [identificar murmúrio vesicular e ruídos adventícios, antes, durante e após intervenção];

- Vigiar ventilação [expansibilidade, tipo, ritmo, amplitude, tempos inspiratórios e

Intervenções de Enfermagem de Reabilitação expiratórios, utilização de músculos acessórios, antes, durante e após intervenção];

- Otimizar a ventilação através da técnica de posicionamento [correção postural, posição de descanso e relaxamento; elevação da cabeceira a $30-45^{\circ}$ ];

- Executar técnicas respiratórias [controlo e dissociação dos tempos respiratórios; expiração com lábios semicerrados; reeducação abdominodiafragmática; reeducação costal bilateral]; - Executar cinesiterapia respiratória [abertura costal global e seletiva à esquerda com foco na inspiração até à capacidade pulmonar total e pausa teleinspiratória];

- Otimizar ventilação através de dispositivos respiratórios [vibração percussiva intrapulmonar, oscilação extratorácica de alta frequência].

Diagnostico de enfermagem: Expetorar ineficaz

Intervenções de Enfermagem de Reabilitação

- Avaliar reflexo da tosse;

- Incentivar a tossir;

- Incentivar a ingestão de líquidos;

- Executar inaloterapia através de inalador;

- Executar cinesiterapia respiratória [abertura costal global e seletiva à esquerda com foco na inspiração até à capacidade pulmonar total e pausa teleinspiratória, vibração percussiva intrapulmonar, oscilação extratorácica de alta frequência];

- Assistir a tossir [tosse assistida, tosse dirigida];

- Vigiar a expetoração.

Diagnóstico de enfermagem: Potencial para melhorar conhecimento sobre técnica da tosse

Intervenções de Enfermagem de Reabilitação
- Avaliar conhecimento sobre técnica da tosse;

- Ensinar sobre técnica da tosse [modulação do fluxo expiratório de baixo a alto volume;

huffing e técnica da expiração forçada];

- Instruir técnica da tosse;

- Treinar técnica da tosse.

Diagnóstico de enfermagem: Potencial para melhorar capacidade para usar técnica da tosse

Intervenções de Enfermagem de Reabilitação
- Avaliar capacidade para usar a técnica da tosse;

- Instruir sobre técnica da tosse [modulação do fluxo expiratório de baixo a alto volume; huffing e técnica da expiração forçada];

- Treinar a técnica da tosse. 


\section{RESULTADOS}

O programa de reeducação funcional respiratória foi concretizado durante cinco dias consecutivos, com duração aproximada de 60 minutos cada sessão.

Perante este caso de uma pessoa portadora de atelectasia obstrutiva por tampão mucoso com fluxo de tosse ineficaz, o processo de reabilitação teve como resultados esperados a diminuição da viscosidade do muco, o aumento do fluxo expiratório, da depuração ciliar e reexpansão pulmonar. Para tal, foi implementada terapia vibrooscilatória intra e extratorácica (com o objetivo de modificar a reologia do muco, induzir a tosse por estimulação mecânica das vias aéreas e promover a reexpansão pulmonar), intercalada com modulação do fluxo expiratório de baixo a alto volume, huffing e técnica da expiração forçada.

A terapia de oscilação extratorácica de alta frequência foi utilizada com recurso a colete vibratório da marca Vest ${ }^{\circledR}$ durante três sessões de 16 minutos/cada. O protocolo usado foi diariamente adaptado com a definição dos parâmetros segundo a tolerância da pessoa, sendo descontinuada quando se constatou diminuição da viscoelasticidade do muco e aumento do fluxo de tosse, como se pode verificar no Quadro 3.

A terapia de vibração percussiva intrapulmonar de alta frequência foi utilizada durante os 5 dias do programa com recurso ao dispositivo Metaneb $^{\circledR}$, tendo o protocolo usado sido igualmente diariamente adaptado segundo a tolerância da pessoa, diminuição da viscoelasticidade do muco e aumento da expansibilidade pulmonar, como se constata no Quadro 4.

Quadro 3 - Terapia de oscilação extratorácica de alta frequência

\begin{tabular}{|c|c|c|c|c|c|c|c|c|c|c|c|c|}
\hline \multicolumn{10}{|c|}{ Oscilação extratorácica de alta frequência (Vest $®$ ) } \\
\hline Parâmetros & \multicolumn{3}{|c|}{$02 / 08 / 2021$} & \multicolumn{3}{c|}{$03 / 08 / 2021$} & \multicolumn{4}{c|}{$04 / 08 / 2021$} \\
\hline Oscilação $(\mathrm{Hz})$ & 7 & 9 & 11 & 11 & 7 & 9 & 11 & 14 & 7 & 10 & 12 & 15 \\
\hline Pressão (nível) & 4 & 4 & 5 & 5 & 4 & 5 & 5 & 6 & 4 & 6 & 6 & 6 \\
\hline Duração (min) & 4 & 4 & 4 & 4 & 4 & 4 & 4 & 4 & 4 & 4 & 4 & 4 \\
\hline
\end{tabular}

Quadro 4 - Terapia de vibração percussiva intrapulmonar de alta frequência

\begin{tabular}{|c|c|c|c|c|c|c|c|c|c|c|c|}
\hline \multicolumn{12}{|c|}{ Vibração percussiva intrapulmonar (Metaneb®) } \\
\hline \multicolumn{2}{|r|}{ Parâmetros } & \multicolumn{2}{|c|}{$02 / 08 / 2021$} & \multicolumn{2}{|c|}{$03 / 08 / 2021$} & \multicolumn{2}{|c|}{$04 / 08 / 2021$} & \multicolumn{2}{|c|}{$05 / 08 / 2021$} & \multicolumn{2}{|c|}{$06 / 08 / 2021$} \\
\hline \multirow{2}{*}{$\begin{array}{l}\text { 운 } \\
\text { 논 }\end{array}$} & \multirow{2}{*}{$\begin{array}{l}\text { Oscilação (Hz) } \\
\text { Duração (min) }\end{array}$} & BF & $\mathrm{AF}$ & $\mathrm{BF}$ & $\mathrm{AF}$ & $\mathrm{BF}$ & $\mathrm{AF}$ & $\mathrm{BF}$ & $\mathrm{AF}$ & $\mathrm{BF}$ & $\mathrm{AF}$ \\
\hline & & 4 & 2 & 4 & 2 & 4 & 2 & 3 & 3 & 3 & 3 \\
\hline \multirow{2}{*}{ 岂 } & \multirow{2}{*}{$\begin{array}{c}\text { Pressão }\left(\mathrm{cmH}_{2} \mathrm{O}\right) \\
\text { Duração (min) }\end{array}$} & \multicolumn{2}{|c|}{10} & \multicolumn{2}{|c|}{10} & \multicolumn{2}{|c|}{15} & \multicolumn{2}{|c|}{20} & \multicolumn{2}{|c|}{20} \\
\hline & & \multicolumn{2}{|c|}{6} & \multicolumn{2}{|c|}{6} & \multicolumn{2}{|c|}{4,5} & \multicolumn{2}{|c|}{4,5} & \multicolumn{2}{|c|}{3} \\
\hline \multicolumn{2}{|c|}{ Resistência (nível) } & \multicolumn{2}{|c|}{ Baixa/Média } & \multicolumn{2}{|c|}{ Média } & \multicolumn{2}{|c|}{ Média } & \multicolumn{2}{|c|}{ Média } & \multicolumn{2}{|c|}{ Média } \\
\hline & $\begin{array}{c}\text { Aerossol } \\
\text { Duração (min) }\end{array}$ & \multicolumn{2}{|c|}{6} & \multicolumn{2}{|c|}{6} & \multicolumn{2}{|c|}{6} & \multicolumn{2}{|c|}{6} & \multicolumn{2}{|c|}{6} \\
\hline \multicolumn{2}{|c|}{$\begin{array}{l}\text { Duração total da sessão } \\
\text { (min) }\end{array}$} & \multicolumn{2}{|c|}{18} & \multicolumn{2}{|c|}{18} & \multicolumn{2}{|c|}{16,5} & \multicolumn{2}{|c|}{16,5} & \multicolumn{2}{|c|}{15} \\
\hline
\end{tabular}

Legenda: CHFO - oscilação contínua de alta frequência; CPEP - pressão expiratória positiva contínua; BF - baixa frequência; AF alta frequência;

No decurso do processo de reabilitação foram sistematicamente avaliados os diferentes parâmetros em estudo. No início a respiração caraterizava-se por ser mista e irregular (abdominodiafragmática ao $3^{\circ}$ dia), com amplitude diminuída (normal ao $4^{\circ}$ dia) e com recrutamento dos músculos acessórios até ao $3^{\circ}$ dia, verificando-se uma melhoria gradual do padrão respiratório. A alta viscoelasticidade do muco condicionava um baixo e ineficaz fluxo de tosse, verificando-se uma melhoria das características do muco ao $3^{\circ}$ dia de tratamento em virtude da aerossolterapia, terapia vibro-oscilatória e aquisição da capacidade para usar a técnica da tosse, como se observa no Quadro 5. 
Quadro 5-Resultados da avaliação por inspeção

\begin{tabular}{|c|c|c|c|c|c|}
\hline Parâmetros & $02 / 08 / 2021$ & $03 / 08 / 2021$ & $04 / 08 / 2021$ & $05 / 08 / 2021$ & $06 / 08 / 2021$ \\
\hline Tipo de respiração & Mista & Mista & $\begin{array}{c}\text { Abdomino- } \\
\text { diafragmática }\end{array}$ & $\begin{array}{c}\text { Abdomino- } \\
\text { diafragmática }\end{array}$ & $\begin{array}{l}\text { Abdomino- } \\
\text { diafragmática }\end{array}$ \\
\hline Ritmo & Irregular & Regular & Regular & Regular & Regular \\
\hline Amplitude & Diminuída & Diminuída & Diminuída & Normal & Normal \\
\hline Músculos Acessórios & $\operatorname{Sim}$ & $\operatorname{Sim}$ & Não & Não & Não \\
\hline $\begin{array}{c}\text { Muco } \\
\text { Cor } \\
\text { Viscoelasticidade }\end{array}$ & $\begin{array}{l}\text { Presente } \\
\text { Amarela } \\
\text { Alta }\end{array}$ & $\begin{array}{l}\text { Presente } \\
\text { Amarela } \\
\text { Alta }\end{array}$ & $\begin{array}{l}\text { Presente } \\
\text { Amarela } \\
\text { Média }\end{array}$ & $\begin{array}{l}\text { Presente } \\
\text { Amarela } \\
\text { Baixa }\end{array}$ & $\begin{array}{c}\text { Presente } \\
\text { Branca } \\
\text { Baixa }\end{array}$ \\
\hline Tosse & Ineficaz & Ineficaz & Pouco eficaz & Pouco eficaz & Pouco eficaz \\
\hline Aerossolterapia & Sim & Sim & Sim & Sim & Sim \\
\hline Oxigenoterapia (L/min) & 2 & 2 & 2 & 2 & 2 \\
\hline
\end{tabular}

Os cuidados de enfermagem de reabilitação respiratória foram orientados mediante a avaliação periódica da auscultação pulmonar de forma a titular os parâmetros das terapias instituídas. No pulmão direito constatou-se inicialmente uma diminuição global do murmúrio vesicular (MV) com presença de fervores crepitantes, verificando-se uma recuperação total ao 4 ㅇ dia de tratamento. No pulmão esquerdo, a atelectasia total condicionava uma ausência do MV na 1a sessão, o qual foi progressivamente recuperado e mantido nos terços superior e médio até à 5a sessão, em linha com o aumento do fluxo de tosse a maior expansibilidade pulmonar, verificando-se esses aspetos no Quadro 6.

Quadro 6- Resultados da avaliação por auscultação pulmonar anterior

\begin{tabular}{|c|c|c|c|c|c|c|c|c|c|c|c|}
\hline & Data & \multicolumn{2}{|c|}{$02 / 08 / 2021$} & \multicolumn{2}{|c|}{$03 / 08 / 2021$} & \multicolumn{2}{|c|}{$04 / 08 / 2021$} & \multicolumn{2}{|c|}{$05 / 08 / 2021$} & \multicolumn{2}{|c|}{$06 / 08 / 2021$} \\
\hline \multicolumn{2}{|c|}{ Bilateralidade pulmonar } & Dta & Esq & Dta & Esq & Dta & Esq & Dta & Esq & Dta & Esq \\
\hline \multirow{3}{*}{$\begin{array}{l}\text { Murmúrio } \\
\text { Vesicular }\end{array}$} & $1 / 3$ superior & $\mathrm{D}$ & A & $D$ & $\mathrm{D}$ & $M$ & $D$ & M & $M$ & $M$ & M \\
\hline & 1/3 médio & $D$ & $A$ & $\mathrm{D}$ & $A$ & M & $\mathrm{D}$ & M & $D$ & $M$ & M \\
\hline & $1 / 3$ inferior & $D$ & $A$ & $\mathrm{D}$ & $A$ & $\mathrm{D}$ & $A$ & M & $\mathrm{D}$ & M & $\mathrm{D}$ \\
\hline \multirow{3}{*}{$\begin{array}{c}\text { Ruídos } \\
\text { Adventícios }\end{array}$} & $1 / 3$ superior & $\mathrm{FC}$ & $A$ & $\mathrm{FC}$ & $\mathrm{FC}$ & $A$ & FC & $A$ & $A$ & $\mathrm{~A}$ & A \\
\hline & $1 / 3$ médio & $\mathrm{FC}$ & $A$ & FC & $A$ & $A$ & $\mathrm{FC}$ & $A$ & $A$ & $A$ & $A$ \\
\hline & $1 / 3$ inferior & FC & $A$ & FC & A & $\mathrm{FC}$ & $A$ & $A$ & $\mathrm{FC}$ & $A$ & $\mathrm{FC}$ \\
\hline
\end{tabular}

Legenda: Dta - Direita; Esq - Esquerda; M - Mantido; D - Diminuído; A - Ausente; FC - Fervores crepitantes

A evolução dos parâmetros vitais traduz a eficácia do tratamento instituído, pela melhoria da $\mathrm{SpO}_{2}$ (93\% vs $97 \%$ ) e redução da frequência respiratória (20 vs 15 ciclos/min) da primeira para a última sessão, com o mesmo aporte de oxigénio administrado, como explanado no Quadro 7.

Quadro 7- Avaliação dos sinais vitais e oximetria periférica

\begin{tabular}{|c|c|c|c|c|c|}
\hline Parâmetros & $02 / 08 / 2021$ & $03 / 08 / 2021$ & $04 / 08 / 2021$ & $05 / 08 / 2021$ & $06 / 08 / 2021$ \\
\hline Tensão Arterial (mmHg) & $95 / 66$ & $90 / 59$ & $97 / 56$ & $118 / 83$ & $119 / 79$ \\
\hline $\begin{array}{c}\text { Frequência Cardíaca (bpm) } \\
\text { Frequência Respiratória } \\
\text { (ciclos/min) }\end{array}$ & 80 & 91 & 84 & 83 & 79 \\
\hline Temperatura ( $\left.{ }^{\circ} \mathrm{C}\right)$ & 20 & 28 & 22 & 36,8 & 36,1 \\
\hline Oximetria (\%) & 93,0 & 36,2 & 36,1 & 95 & 97 \\
\hline
\end{tabular}


Pela análise dos valores obtidos nas gasometrias arteriais, pode verificar-se que as 3 primeiras sessões são marcadas por hipoxémia considerável, que não teve repercussões observáveis no registo de $\mathrm{SpO}_{2}$ nem da frequência cardíaca. Estes registos foram obtidos nas sessões realizadas com maior intervalo de tempo. Na última sessão existe uma estabilização geral dos parâmetros dentro dos limites estabelecidos pela bibliografia e uma ligeira melhoria da hipoxémia. Ainda decorrente dos resultados advindos da gasometria arterial, elucidados no Quadro 8, pode observar-se uma evidente melhoria da oxigenação, comprovada pelo aumento significativo da $\mathrm{SpO}_{2}$ sob a mesma $\mathrm{FiO}_{2}$. Do ponto de vista imagiológico, através da análise das telerradiografias, explanadas na Figura 1, os resultados são demonstrativos da evolução, pois no dia 02/08/21 verificava-se uma hipotransparência pulmonar em praticamente todo o campo pulmonar esquerdo com desvio do mesmo, sugestivo de atelectasia, ao passo que no dia 06/08/21 era notória uma resolução praticamente completa da atelectasia pulmonar esquerda, com o mediastino centrado, com uma ligeira opacidade no ângulo costofrénico.

Quadro 8- Resultados da Gasometria arterial

\begin{tabular}{|c|c|c|c|c|c|c|c|}
\hline Data & $\begin{array}{c}\mathrm{O}_{2} \\
(\mathrm{~L} / \mathrm{min})\end{array}$ & $\mathrm{pH}$ & $\begin{array}{c}\mathrm{pCO}_{2} \\
(\mathrm{mmHg})\end{array}$ & $\begin{array}{c}\mathrm{pO}_{2} \\
(\mathrm{mmHg})\end{array}$ & $\begin{array}{c}\text { Lact } \\
(\mathrm{mmol} / \mathrm{L})\end{array}$ & $\begin{array}{c}\mathrm{SpO}_{2} \\
(\%)\end{array}$ & $\begin{array}{c}\mathrm{HCO}_{3} \\
(\mathrm{mmol} / \mathrm{L})\end{array}$ \\
\hline $03 / 08 / 2021$ & $2 \mathrm{~L} / \mathrm{min}$ & 7.46 & 51 & 63 & 2.3 & $93 \%$ & 36.3 \\
\hline $06 / 08 / 2021$ & $2 \mathrm{~L} / \mathrm{min}$ & 7.44 & 66 & 106 & 1,7 & $98 \%$ & 31.8 \\
\hline $09 / 08 / 2021$ & $1 \mathrm{~L} / \mathrm{min}$ & 7.44 & 66 & 96 & 1,5 & $97 \%$ & 44.8 \\
\hline $16 / 08 / 2021$ & $\cdots$ & 7.44 & 57 & 93 & 1,9 & $98 \%$ & 38,7 \\
\hline
\end{tabular}

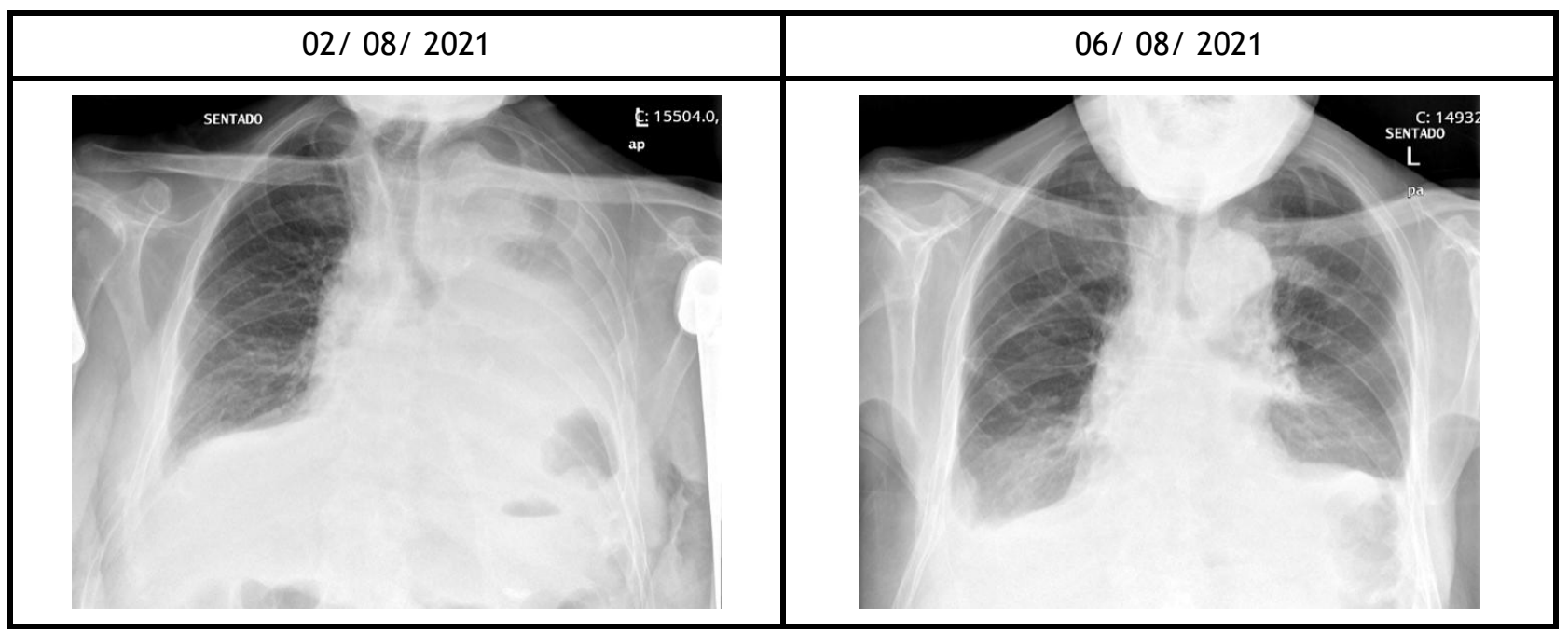

Figura 1- Telerradiografia do tórax

\section{DISCUSSÃO}

Perante o caso clínico de uma pessoa portadora de atelectasia pulmonar obstrutiva secundária a tampão mucoso, foi delineado e implementado um programa de enfermagem de reabilitação alicerçado em evidência científica e harmonizado com a condição clínica, permitindo, desta forma, uma abordagem de caráter particular, multidisciplinar, com um cuidado centrado na pessoa, como descrito na literatura ${ }^{(6,10)}$.

O método exposto e os resultados alcançados permitem expressar que os cuidados de enfermagem de reabilitação representam um papel preponderante na recuperação e a efetividade das intervenções no controlo e redução da dispneia da pessoa, potenciando a qualidade de vida da pessoa e a rapidez de recuperação ${ }^{(22)}$.

O programa de reabilitação na pessoa portadora de atelectasia pulmonar obstrutiva secundária a tampão mucoso deverá incidir sobre a ventilação, o expetorar e a capacidade e conhecimento sobre a técnica da tosse, pelo, que após a avaliação inicial de enfermagem de reabilitação foram identificados os focos e diagnósticos e delineadas intervenções de enfermagem de reabilitação assentes em: reeducação funcional respiratória, baseada nas técnicas de controlo e dissociação dos tempos respiratórios, expiração com lábios semicerrados, reeducação abdominodiafragmática, reeducação costal bilateral, abertura costal global e seletiva à esquerda com foco na inspiração até à capacidade pulmonar total e pausa teleinspiratória, vibração percussiva intrapulmonar e oscilação 
extratorácica de alta frequência. Perante o expetorar comprometido e o potencial de melhoria do conhecimento e da capacidade da técnica da tosse, foi realizado ensino, instrução e treino da técnica da tosse, com modulação do fluxo expiratório de baixo a alto volume, huffing e técnica da expiração forçada, tendo por base as intervenções efetivas descritas na literatura. Nesse sentido, o plano de intervenção concretizado teve como fundamento a evidência científica, sendo demonstrativo que uma prática baseada na evidência pode "melhorar os resultados do cuidado na pessoa ou criar diretrizes para a prática clínica"(23).

A terapia vibro-oscilatória tem como objetivo promover a limpeza das vias aéreas, o recrutamento alveolar e melhorar as trocas gasosas. É uma técnica bem tolerada, não havendo efeitos adversos descritos na literatura ${ }^{(11)}$.

A implementação do plano de intervenção de enfermagem de reabilitação proporcionou ganhos em saúde na pessoa que se podem objetivar, nomeadamente na redução e controlo da dispneia, constatando-se igualmente melhoria das características da respiração, do muco, da auscultação pulmonar e analiticamente, como igualmente descrito na literatura científica ${ }^{(3,7)}$. Estas melhorias foram obtidas de forma gradual e progressiva e são demonstrativas de um cuidado centrado na pessoa, onde o cuidar pode ser conceptualizado como uma "intervenção terapêutica focada em ações de cuidado que atendam às necessidades da pessoa e que resultem em melhorias positivas”(24).

Apesar das limitações inerentes à condição clínica e particularidades da pessoa, é viável atestar que as intervenções do EEER são consideradas indispensáveis para alcançar os objetivos propostos e para a melhoria global da função respiratória, controlo e gestão dos sintomas (dispneia). Nesse sentido, é tácito que o EEER é detentor de conhecimentos específicos e fundamentados para avaliar, prescrever e implementar intervenções, concretizando o Processo de Enfermagem na pessoa com atelectasia.

\section{CONSIDERAÇÕES FINAIS}

Este relato de caso possibilitou evidenciar a relevância e efetividade das intervenções de enfermagem de reabilitação implementadas pelo EEER na pessoa portadora de atelectasia pulmonar.

Ao longo da implementação do programa foi necessária flexibilidade no sentido de um ajustamento, tanto das intervenções a implementar como dos objetivos previamente definidos. 0 cuidado centrado na pessoa surge como estrutura conceptual onde é suportada uma interação entre o enfermeiro e a pessoa e o cuidar como intervenção terapêutica que acarreta a possibilidade de resultados positivos para a pessoa.

O progresso da condição clínica da pessoa foi, de uma forma genérica, patente, ainda que as evidências se tenham revelado mais evidentes em determinados diagnósticos que culminaram na gestão de sintomas, como a dispneia. Nesse sentido, este relato de caso torna-se uma janela de oportunidade no sentido de evidenciar que o EEER é um dos elementos dinâmicos da equipa multidisciplinar promotor de uma recuperação mais célere e consequentemente uma gestão eficaz dos sintomas e concludentemente melhoria na qualidade de vida da pessoa, que se repercutiu numa alta precoce, reinserção na comunidade e diminuição do número de dias de internamento.

Estes dados reiteram a importância da intervenção do EEER junto da pessoa com alterações da função respiratória, através da implementação de um programa de reeducação funcional respiratória, de capacitação do utente e de gestão dos sintomas.

A técnica vibro-oscilatória aplicada neste caso concreto a um paciente com o diagnóstico de atelectasia pulmonar verificou-se eficaz. A utilização desta técnica deve ser ponderada, após consideração dos seus riscos/benefícios e mediante as características individuais da pessoa com necessidades de reabilitação funcional respiratória por Enfermagem de Reabilitação.

\section{REFERÊNCIAS BILIOGRÁFICAS}

1. Duggan M, Kavanagh B. Perioperative modifications of respiratory function. Best Pract Res Clin Anaesthesiol. 2010 Jun;24(2):145-55. Available from: https://doi.org/10.1016/j.bpa.2009.12.001

2. Klein J, Rosado-de-Christenson M. A Systematic Approach to Chest Radiographic Analysis. In: Hodler J, Kubik-Huch R, von Schulthess G, editors. Diseases of the Chest, Breast, Heart and Vessels. Springer; 2019.

3. Grott K, Dunlap J. Atelectasis. In: StatPearls. Treasure Island: StatPearls Publishing; 2021.

4. Rama-Maceiras P. [Peri-operative atelectasis and alveolar recruitment manoeuvres]. Arch Bronconeumol. 2010 Jun;46(6):317-24. Available from: https://doi.org/10.1016/j.arbres.2009.10.010

5. Schindler M. Treatment of atelectasis: where is the evidence? Crit Care. 2005/07/07. 2005 Aug;9(4):341-2. Available from: https://doi.org/10.1186/cc3766

6. Restrepo R, Braverman J. Current challenges in the recognition, prevention and treatment of perioperative pulmonary atelectasis. Expert Rev Respir Med. 2015 Feb;9(1):97-107. Available from: https://doi.org/10.1586/17476348.2015.996134

7. Odor P, Bampoe S, Gilhooly D, Creagh-Brown B, Moonesinghe S. Perioperative interventions for prevention of postoperative pulmonary complications: systematic review and meta-analysis. BMJ. 2020 Mar;368:m540. Available from: https://doi.org/10.1136/bmj.m540

8. Button B, Button B. Structure and function of the mucus clearance system of the lung. Cold Spring Harb Perspect Med. 2013 Aug 1;3(8):a009720. Available from: https://doi.org/10.1101/cshperspect.a009720

9. Mcllwaine M, Bradley J, Elborn J, Moran F. Personalising airway clearance in chronic lung disease. Eur Respir Rev. 2017 Mar 31;26(143):160086. Available from: https://doi.org/10.1183/16000617.0086-2016 
10. Chalmers J, Aliberti S, Blasi F. State of the art review: management of bronchiectasis in adults. Eur Respir J. 2015 Mar 18;ERJ-01191-2014. Available from: https: //doi.org/10.1183/09031936.00119114

11. Reychler G, Coppens T, Leonard A, Palem A, Lebecque P. Mucoviscidose: les techniques instrumentales de désencombrement des voies aériennes. Rev Mal Respir. 2012 Feb 1;29:128-137. Available from: https://doi.org/10.1016/j.rmr.2011.11.013

12. Pieterse A, Hanekom S. Criteria for enhancing mucus transport: a systematic scoping review. Multidiscip Respir Med. $2018 ; 13(1): 22$. Available from: https://doi.org/10.1186/s40248-018-0127-6

13. Kim V, Garfield J, Grabianowski C, Krahnke J, Gaughan J, Jacobs M, et al. The effect of chronic sputum production on respiratory symptoms in severe COPD. COPD. 2011 Apr;8(2):114-20. Available from: https://doi.org/10.3109/15412555.2011.558546

14. Belli S, Prince I, Savio G, Paracchini E, Cattaneo D, Bianchi M, et al. Airway Clearance Techniques: The Right Choice for the Right Patient. Front Med. 2021;8:544826. Available from: https://doi.org/10.3389/fmed.2021.544826

15. Darbee J, Kanga J, Ohtake P. Physiologic evidence for high-frequency chest wall oscillation and positive expiratory pressure breathing in hospitalized subjects with cystic fibrosis. Phys Ther. 2005 Dec;85(12):1278-89.

16. Testa A, Galeri S, Villafañe J, Corbellini C, Pillastrini P, Negrini S. Efficacy of short-term intrapulmonary percussive ventilation in patients with chronic obstructive pulmonary disease. Disabil Rehabil. 2015;37(10):899-903. Available from: https: / /doi.org/10.3109/09638288.2014.948130

17. Riffard G, Toussaint $M$. Ventilation à percussions intrapulmonaires: fonctionnement et modalités de réglage. Rev Mal Respir. 2012;29(2):347-54. Available from: https://doi.org/https://doi.org/10.1016/j.rmr.2011.12.003

18. Reychler G, Debier E, Contal O, Audag N. Intrapulmonary Percussive Ventilation as an Airway Clearance Technique in Subjects With Chronic Obstructive Airway Diseases. Respir Care. 2018 May;63(5):620-31. Available from: https://doi.org/10.4187/respcare.05876

19. Riley D, Barber M, Kienle G, Aronson J, von Schoen-Angerer T, Tugwell P, et al. CARE guidelines for case reports: explanation and elaboration document. J Clin Epidemiol. 2017 Sep;89:218-35. Available from: https://doi.org/10.1016/j.jclinepi.2017.04.026

20. OE. Padrão documental dos cuidados de enfermagem da especialidade de enfermagem de reabilitação 2014 [Internet]. Ordem dos Enfermeiros. Porto; 2014.

21. OE. Guia Orientador de Boa Prática: Reabilitação Respiratória [Internet]. Lisboa; 2018.

22. Doran D, Pringle D. Patient Outcomes as an Accountability. In: D. M. Doran, editor. Nursing Outcomes - The State of the Science. 2003. p. 1 27.

23. Stanik-Hutt J. Translation of Evidence to Improve Clinical Outcomes. In: Translation of Evidence Into Nursing and Healthcare. 2021. Available from: https://doi.org/https://doi.org/10.16309/j.cnki.issn.1007-1776.2003.03.004

24. McCormack B, McCance T. A Theoretical Framework for Person-Centred Nursing. In: Person-Centred Nursing. 2010. p. 26. (Wiley Online Books). Available from: https://doi.org/https://doi.org/10.1002/9781444390506.ch3

\section{DIVULGAÇÕES ÉTICAS}

Todos os autores leram e concordaram com a versão publicada do manuscrito.

Financiamento:

Este trabalho não recebeu nenhuma contribuição financeira ou bolsa.

Comissão de Ética:

Estudo aprovado pela comissão de ética do Centro Hospitalar de Trás-os-Montes e Alto Douro, E.P.E.

Declaração de consentimento informado:

Não se aplica

\section{Agradecimentos:}

Os autores agradecem a todos os que têm colaborado e permitido continuar a investigar nesta área

Conflitos de interesse:

Os autores não declaram nenhum conflito de interesses.

Proveniência e revisão por pares:

Não comissionado; revisto externamente por pares.

(C) Autor (es) (ou seu (s) empregador (es)) e APER/RPER 2022. Reutilização permitida de acordo com CC BY-NC-ND. Nenhuma reutilização comercial. 